\title{
Embolia cutis medicamentosa: an unusual adverse reaction to terlipressin
}

\author{
Polychronis Gatos-Gatopoulos ${ }^{a}$, Stephanos Kostantoudakis ${ }^{b}$, loannis G. Panayiotides ${ }^{b}$, \\ George D. Dimitriadisa, Konstantinos Triantafyllou ${ }^{a}$
}

Medical School, National and Kapodistrian University of Athens, Attikon University Genera Hospital, Athens, Greece

\begin{abstract}
Terlipressin is a synthetic long-acting analog of vasopressin widely used to control variceal bleeding by lowering portal venous pressure. We report an unusual adverse reaction to terlipressin in a 78-year-old patient with esophageal variceal bleeding who developed skin necrosis soon after treatment initiation. Skin biopsy revealed embolia cutis medicamentosa.
\end{abstract}

Keywords Terlipressin, embolia cutis medicamentosa, skin necrosis, Nicolau syndrome, esophageal variceal bleeding

Ann Gastroenterol 2017; 30 (3): 1-4

\section{Introduction}

The initial management of variceal bleeding is vasoactive pharmacological therapy with octreotide, somatostatin, terlipressin, or vasopressin. We report a case of terlipressininduced extensive skin necrosis in a 78 -year-old female with cirrhosis and esophageal variceal bleeding.

\section{Case report}

A 78-year-old Caucasian female presented with hematemesis. She was obese and cirrhotic as a result of non-alcoholic steatohepatitis. Clinical examination revealed confusion, blood pressure $90 / 42 \mathrm{mmHg}$, heart rate 135 beats/min, and respiratory rate 18 breaths/min. Laboratory

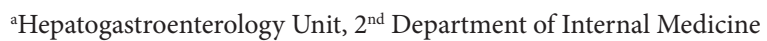
- Propedeutic, Research Institute and Diabetes Center (Polychronis Gatos-Gatopoulos, George D. Dimitriadis, Konstantinos Triantafyllou); ${ }^{b} 2^{\text {nd }}$ Department of Pathology (Stephanos Kostantoudakis, Ioannis G. Panayiotides), Medical School, National and Kapodistrian University of Athens, Attikon University General Hospital, Athens, Greece

Conflict of Interest: None

Correspondence to: Dr. Konstantinos Triantafyllou, MD, PhD, FEBGH, Associate Professor of Gastroenterology, Hepatogastroenterology Unit, $2^{\text {nd }}$ Dept. of Internal Medicine, Research Institute and Diabetes Center, Medical School, National and Kapodistrian University of Athens, Attikon University Hospital, 1 Rimini Street, 12464 Athens, Greece, Tel.: +30 210 5832090, Fax: +30 2105326422 ,

e-mail: ktriant@med.uoa.gr

Received 22 March 2017; accepted 18 April 2017; published online 11 May 2017

DOI: https://doi.org/10.20524/aog.2017.0158 findings included anemia, with hemoglobin $5.2 \mathrm{~g} / \mathrm{dL}$ (normal range 12.0-15.0 g/dL), international normalized ratio 1.56 (normal range 0.90-1.20), blood glucose $571 \mathrm{mg} / \mathrm{dL}$ (normal range 74-106 mg/dL) and blood creatinine $1.3 \mathrm{mg} / \mathrm{dL}$ (normal range 0.5-0.9 mg/dL). Bilirubin, transaminases and cholestatic enzymes were within their normal range.

Variceal bleeding was suspected and the patient received two units of red blood cells, terlipressin $2 \mathrm{mg}$ (Glypressin ${ }^{\circledast}$, Ferring Pharmaceutical Hellas, Greece) and ceftriaxone $1 \mathrm{~g}$ iv (Rocephin ${ }^{\circledast}$, Roche Hellas, Greece). Upper gastrointestinal endoscopy under anesthesia identified actively bleeding esophageal varices, ligated endoscopically.

Two hours after the third $2 \mathrm{mg}$ dose of terlipressin iv (doses given every $4 \mathrm{~h}$ ), the patient developed purpuric skin lesions on her left arm (Fig. 1A), both breasts (Fig. 1C) and lower abdomen (Fig. 1B, D). Terlipressin treatment was terminated. The lesions evolved rapidly (Fig. 2) to necrosis and a cutaneous biopsy performed $48 \mathrm{~h}$ after the start of treatment led to the diagnosis of embolia cutis medicamentosa (ECM) or Nicolau syndrome (Fig. 3). The patient died from sepsis 8 days after her admission.

\section{Discussion}

Terlipressin doses of 1-2 mg iv, bolus and every 4-6 h thereafter, effectively reduce the portal venous pressure and induce marked splanchnic vasoconstriction. Common adverse effects of terlipressin include headache, bradycardia, peripheral vasoconstriction, peripheral ischemia, facial pallor, hypertension, transient abdominal cramps and transient diarrhea, while skin necrosis is reported as an adverse reaction of unknown frequency.

ECM, also known as Nicolau syndrome or livedoid dermatitis, is a rare cutaneous adverse reaction to certain 


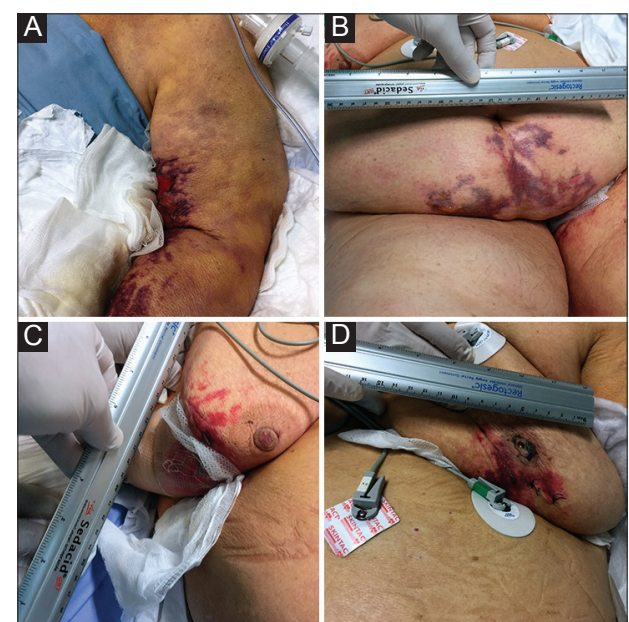

Figure 1 Fourteen hours after initiation of terlipressin treatment skin purpuric lesions developed on the (A) left arm, (B) lower abdomen and $(\mathrm{C}, \mathrm{D})$ breasts
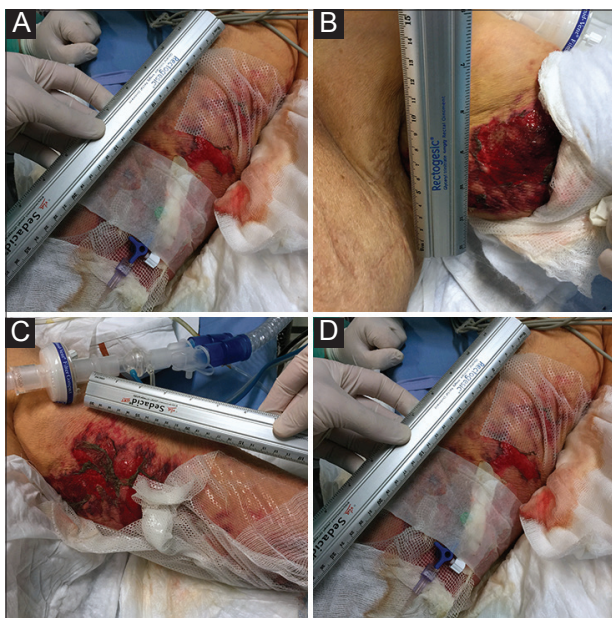

Figure 2 The evolution of skin lesions on the patient's left arm: (A) $24 \mathrm{~h}$ following initiation of terlipressin treatment; (B, C, D) $36 \mathrm{~h}$ following treatment initiation

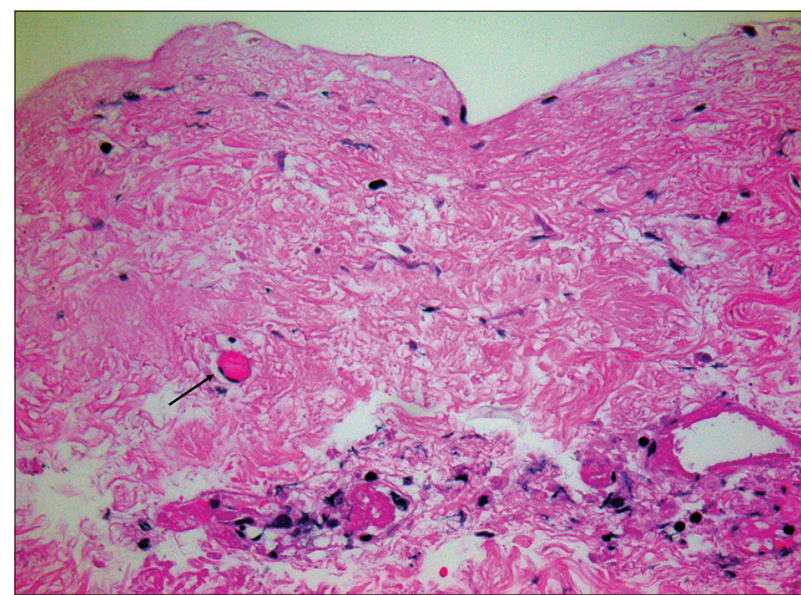

Figure 3 Histology of a cutaneous specimen that disclosed complete absence of the epidermis, as well as the presence of a few fibrin plugs within dermal capillaries (arrow), with no essential inflammation and IgG, IgA, IgM or C3 complement immunohistochemical component medications, such as penicillins, non-steroidal antiinflammatory drugs, local anesthetics and corticosteroids. It is characterized clinically by severe pain immediately after the injection, followed by an erythematous, reticular patch that may result in a necrotic ulcer and scarring. Histopathological examination reveals necrosis of the epidermis, dermis, subcutaneous tissue and muscle, with focal thrombosis of small and medium blood vessels.

A PubMed search using the terms "terlipressin", "skin necrosis", "skin ischemia", "embolia cutis medicamentosa" and "Nicolau syndrome" returned 21 reports of skin necrosis in patients treated with terlipressin, either for variceal bleeding or for hepatorenal syndrome. In addition, the pharmacovigilance department of Ferring Pharmaceutical Hellas, the local terlipressin distributor, referred us to a case of an alcoholic patient who experienced extensive epidermal necrosis following treatment with terlipressin for "stomach bleeding"; however, this case could not be matched with the aforementioned cases because additional patient data were lacking.

Among the reported cases with histological confirmation of terlipressin-induced skin necrosis $(n=14)$, a diagnosis compatible with Nicolau syndrome was made in 6 [1-5] (Table 1), while in the rest no capillary emboli were detected. It is difficult to clarify whether these two distinct histologic types are associated with different mechanisms or stages of terlipressin's action in the skin capillaries, with different affected skin areas (corpus vs. extremities), with the coadministration of other medication, or with the patients' background.

More specifically, our patient developed skin lesions $14 \mathrm{~h}$ after terlipressin treatment initiation, whereas in all but one (time lag not specified, no histology available [6]) of the cases found in PubMed the lesions developed up to $264 \mathrm{~h}$ later. Moreover, in the 2 cases where lesions occurred within $24 \mathrm{~h}$ following treatment initiation [1,4], ECM was detected. Although ischemic skin lesions related to terlipressin administration usually affect the extremities (fingers, scrotum, lower legs and toes), our patient's extremities were spared.

One of the confounding factors in the causative relation leading to adverse events after a treatment is co-administered medication. Antibiotic administration has been related to ECM, and our patient received ceftriaxone for infection prophylaxis, as per guidelines. There are no literature reports of ECM associated with ceftriaxone use, and a safety search of the Roche Medical Information Department following our request detected no such signal. However, colleagues from India have reported Nicolau syndrome (without histological confirmation) in a child treated with ceftriaxone for Dengue fever [7]. Finally, there is no reported association of ECM with the use of propofol (Propofol ${ }^{\oplus}$, Fresenius Kabi Hellas, Greece) or midazolam (Midazolam/B. Braun ${ }^{\oplus}$, Bioser, Greece), which were administered to our patient during endoscopy.

The majority of cases of skin necrosis associated with terlipressin use occurred in alcoholic cirrhotics suffering from hepatorenal syndrome or variceal bleeding. Several predisposing factors for skin necrosis in these patients have been reported, including obesity [3,5], hypovolemia [4,5], 


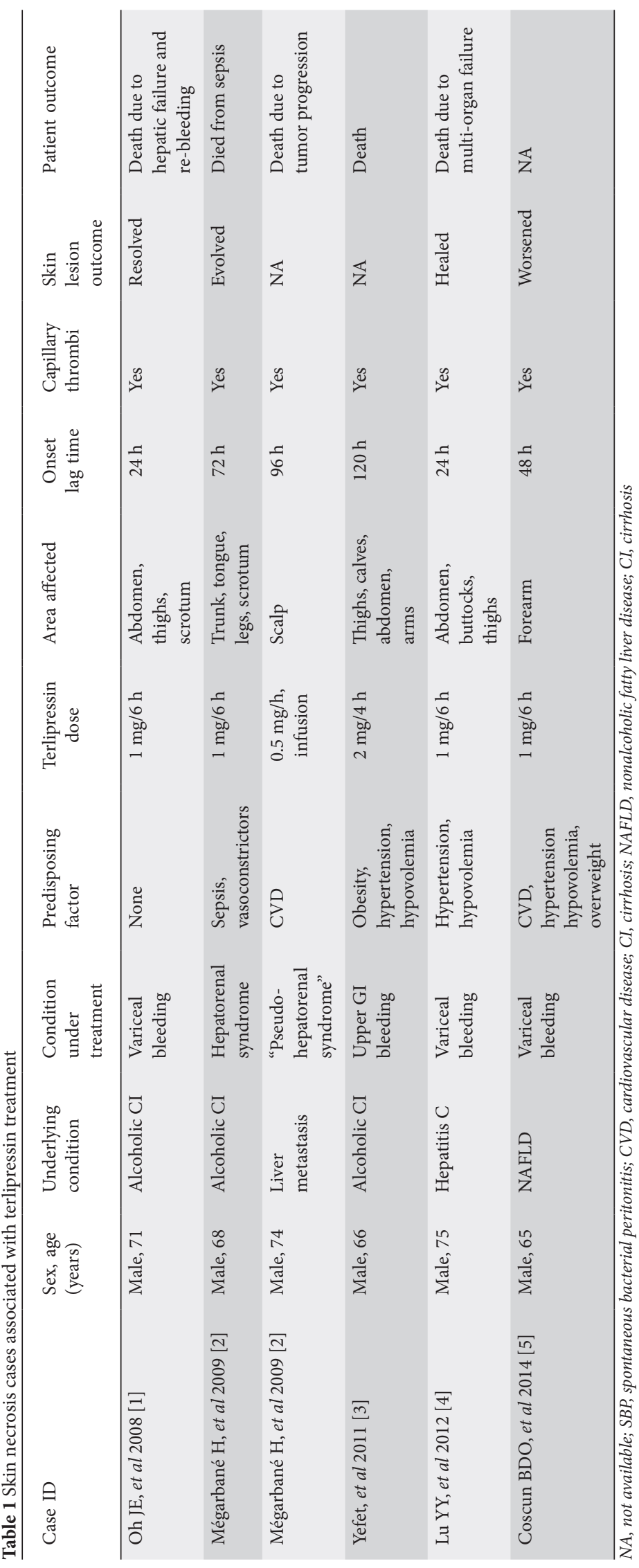


use of vasoconstrictive agents (apart from terlipressin) [2], ischemic vascular disease [2,5] and sepsis [2]. Our patient was non-alcoholic, obese, and hypovolemic at presentation. Although our histology findings were not associated with Staphylococcus capitis skin infection, the possibility of this microbe being implicated in the pathogenesis and/or evolution of ECM cannot be firmly excluded.

The vasopressin analog terlipressin releases von Willebrand factor via activation of $\mathrm{V}_{2}$ receptors in endothelial cells and enhances platelet aggregation. This may lead to capillary thrombosis, especially under intense or prolonged peripheral vasoconstriction [8], which is the case in hepatorenal syndrome and in severe variceal bleeding.

Based on its pharmacology and mechanism of action, literature reports, histological findings and chronological association, we conclude that terlipressin may cause acuteonset ECM with extensive ischemic skin necrosis. It remains to be proven whether ECM is a different entity than skin necrosis without capillary thrombi. Moreover, we cannot exclude the possibility that specific patient background, co-medication and skin infections might also have been involved in the evolution of this rare adverse drug reaction.

\section{References}

1. Oh JE, Ha JS, Cho DH, Yu GJ, Shim SG. A case of ischemic skin necrosis after glypressin therapy in liver cirrhosis. Korean $J$ Gastroenterol 2008;51:381-384.

2. Mégarbané $H$, Barete $S$, Khosrotehrani K, et al. Two observations raising questions about risk factors of cutaneous necrosis induced by terlipressin (Glypressin). Dermatology 2009;218:334-337.

3. Yefet E, Gershovich M, Farber E, Soboh S. Extensive epidermal necrosis due to terlipressin. Isr Med Assoc J 2011;13:180-181.

4. Lu YY, Wei KC, Wu CS. Terlipressin-induced extensive skin necrosis: A case report and published work review. J Dermatol 2012;39:866-868.

5. Ozel Coskun BD, Karaman A, Gorkem H, Buğday I, Poyrazoğlu OK, Senel F. Terlipressin-induced ischemic skin necrosis: A rare association. Am J Case Rep 2014;15:476-479.

6. Donnellan F, Cullen G, Hegarty JE, McCormick PA. Ischaemic complications of Glypressin in liver disease: A case series. Br J Clin Pharmacol 2007;64:550-552.

7. Srivastava P, Someshwar S, Jerajani H. Nicolau's Syndrome. Indian Pediatr 2105;52:356.

8. Kam PCA, Williams S, Yoong FFY. Vasopressin and terlipressin: Pharmacology and its clinical relevance. Anaesthesia 2004;59:993-1001. 